\title{
Endoscopic surgical treatment of Cushing's disease: A single-center experience of cauterization of peritumoral tissues
}

\author{
SONGYU CHEN ${ }^{1-3}$, SHUJUN XU ${ }^{2,3}$, FUXIN LIN $^{2-4}$, XIN ZHANG $^{2,3}$, FUQIANG LIU ${ }^{5}$, \\ MING DONG ${ }^{5}$, XINGANG $\mathrm{LI}^{2,3}$ and XIANGYU MA ${ }^{2,3}$ \\ ${ }^{1}$ Department of Neurosurgery, Shanghai Tenth People's Hospital, Tongji University, Shanghai 200072; \\ ${ }^{2}$ Department of Neurosurgery, Qilu Hospital of Shandong University; ${ }^{3}$ Brain Science Research Institute, \\ Shandong University, Jinan, Shandong 250012; ${ }^{4}$ Department of Neurosurgery, The First Affiliated Hospital of \\ Fujian Medical University, Fujian Medical University, Fuzhou, Fujian 350004; ${ }^{5}$ Department of \\ Endocrinology, Qilu Hospital of Shandong University, Jinan, Shandong 250012, P.R. China
}

Received May 1, 2018; Accepted September 24, 2018

DOI: $10.3892 /$ etm.2019.8075

\begin{abstract}
Endoscopic transsphenoidal surgery is a form of treatment for Cushing's disease that is initially preferred compared with other types of treatment. Peritumoral tissue is inactivated by cauterization if a clear border of the lesion cannot be identified. In order to compare the surgical outcomes and post-operative complications between patients who underwent inactivation (cauterization of peritumoral tissues is referred to as inactivation) and those who did not, the medical records of patients treated between January 2010 and June 2016 were retrospectively reviewed. Furthermore, the results of conventional examinations performed in order to diagnose and locate the tumors, including neuroimaging, high-dose dexamethasone suppression tests and/or bilateral inferior petrosal sinus sampling, were collected. A total of 79 consecutive patients with Cushing's disease were included in the present analysis. Inactivation of peritumoral tissue had been applied in $35(44.3 \%)$ of the cases. A gross total resection was achieved in $73(92.4 \%)$ of the cases, while partial resection had been performed in the remaining six (7.6\%). Early post-operative endocrinological remission was attained in $71(89.8 \%)$ of the patients. A total of seven cases suffered from hypopituitarism, while cerebrospinal fluid rhinorrhea and brain stem infarction were recorded in four cases and one case, respectively. During the follow-up, the duration of which ranged between 11 and 62 months, recurrence was documented in five patients, as determined by endocrinal examinations. All of the cases that were
\end{abstract}

Correspondence to: Dr Xiangyu Ma, Department of Neurosurgery, Qilu Hospital of Shandong University, 107 Wenhua West Road, Jinan, Shandong 250012, P.R. China

E-mail: qlmaxiangyu@163.com

Key words: endoscopic transsphenoidal surgery, Cushing's disease, pituitary adenoma, adrenocorticotropic hormone, remission, comorbidity initially treated unsuccessfully and those with recurrence achieved endocrinological remission following radiotherapy. Enhanced pituitary magnetic resonance imaging revealed no further enlargement of tumors during follow-up. The extent of endocrinological remission, recurrence and post-operative complications did not significantly differ between patients who underwent inactivation and those who did not. In conclusion, inactivation of peritumoral tissue by cauterization achieved satisfactory results in patients with intricate lesions, however it is comparable to conventional procedures.

\section{Introduction}

Cushing's disease is the most common etiology of endogenous hypercortisolism, accounting for $\sim 70 \%$ of cases $(1,2)$. Patients with this disease suffer from excess adrenocorticotropic hormone (ACTH) secretion from the pituitary adenoma cells. Cardiovascular diseases, refractory diabetes mellitus, as well as infections are the major causes of mortality. During endocrinological remission, the risk of death from cardiovascular events may still be higher than that in the general population $(1,2)$.

In 1969, Hardy developed the endoscopic transsphenoidal surgery (ETS) for pituitary ACTH adenoma with the assistance of micro-neurosurgical techniques. Since then, the transsphenoidal approach has been regarded as the optimal method for the treatment of pituitary ACTH adenoma (3-5). The ever-growing clinical implementation of ETS resulted in encouraging outcomes (remission rates of $\sim 80 \%$ ) (6-9). However, $>80 \%$ of ACTH pituitary adenomas are microadenomas, and for those, magnetic resonance imaging (MRI) may only provide normal scans in $50 \%$ of the cases examined (10). Recently, the application of ETS in Qilu Hospital of Shandong University (Jinan, China) for the treatment of functional pituitary adenomas has been used to routinely inactivate peritumoral tissue by cauterization if a clear border of lesion cannot be identified. To the best of our knowledge, the current study is the first to demonstrate the aforementioned method being used to inactivate peritumoral tissue. The present study aimed to review and compare 
endocrinological remission, recurrence and post-operative complications in patients who underwent inactivation and those who did not.

\section{Material and methods}

General information. The medical records of 79 consecutive patients with Cushing's disease who were treated at Qilu Hospital (Jinan, China) between January 2010 and June 2016 were reviewed. The cohort comprised 58 women and 21 men, and the age of the subjects ranged between 33 and 61 years, with a median age of 44 years. A total of 67 cases exhibited truncal obesity, 35 cases had purple striae, 9 cases had visual field defects, 61 cases presented with hypertension and 47 cases with diabetes. A total of 18 cases had transient ischemic attacks (defined as at least one attack within 5 years), 7 female patients had irregular menstruation or amenorrhea and two male patients exhibited defective secondary sexual characteristics. None of the patients included received any medical therapy, including ketoconazole, prior to surgery.

Pre-operative endocrine evaluation. All of the 79 patients exhibited elevated serum cortisol, ranging from 22.1 to $43.5 \mu \mathrm{g} / \mathrm{dl}$ with an average of $27.9 \mu \mathrm{g} / \mathrm{dl}$. The serum ACTH level ranged between 26.2 and $59.1 \mathrm{pg} / \mathrm{ml}$, with an average level was $37.2 \mathrm{pg} / \mathrm{ml}$. A total of 16 patients were not responsive in high-dose dexamethasone suppression testing, and 13 patients without visible tumors on routine enhanced MRI underwent bilateral inferior petrosal sinus sampling (BIPSS). BIPSS confirmed the diagnosis of Cushing's syndrome with IPS based on a ratio of ACTH (IPS/peripheral) of $>2.0$. These cases underwent surgery. The erratic availability of corticotropin-releasing hormone $(\mathrm{CRH})$ in China prevented the application of $\mathrm{CRH}$ stimulation tests to the patients. With the exception of four cases with slightly increased prolactin (PRL $<100 \mathrm{ng} / \mathrm{ml}$ ), other pre-operative pituitary hormones were in the normal range among the remaining patients of the cohort.

Neuroimaging. All patients underwent sphenoid sinus computed tomography three-dimensional reconstruction prior to surgery. The results revealed 66 cases of the sellar type and 13 cases of the presellar type. With the exception of two patients who had metal implants due to a previous surgery, enhanced MRI examination was performed in all patients pre-operatively. If the information provided by the routine enhanced MRI was unsatisfactory, pituitary dynamic MRI scanning was performed. Neuroimaging revealed 11 cases of pituitary hyperplasia, 51 cases of microadenomas ( 2 cases of microadenoma were confirmed by operation without MRI examination) and 14 cases of macroadenoma.

Surgical treatment. All of the included patients underwent ETS, as exemplified in the illustrative case presented in Fig. 1. Rigid $0^{\circ}$ and $30^{\circ}$ endoscopes with an external diameter of $4.5 \mathrm{~mm}$ (Aesculap; B. Braun, Melsungen, Germany) were used. Once the pseudocapsule was identified in part of the tumors, complete tumor resection was achieved along that plane, as exemplified in the illustrative case presented in Fig. 2. However, larger tumors without an identifiable pseudocapsule were resected in a piecemeal fashion with the assistance of tumor forceps, angled curette and suction. If the remaining pituitary gland had a diameter of $\geq 8 \mathrm{~mm}$, the peritumoral tissue was inactivated $(11,12)$. Careful inactivation of the tissue was applied through extensive cauterization following the detection of color changes (from off-white to orange), which indicated the presence of peritumoral tissue. With regard to pituitary microadenomas or lesions that were not identifiable by pre-operative imaging, selective exploration of the pituitary was performed according to the pre-operative imaging results and IPSS. During this process, off-white tumor tissues were always identified and removed from the sella. A total of 24 patients in the study group experienced different degrees of rupture of the arachnoid membrane around the diaphragm sellae. Intra-operatively, autologous fat was harvested in order to fill the intrasellar region and reconstruct the sellar floor. Hematoxylin and eosin stains, and comprehensive immunohistochemical analysis (13) on several parameters, such as ACTH, were performed for all specimens.

Post-operative endocrine evaluation. Normal fasting plasma cortisol and ACTH levels within the first week following surgery were considered to indicate early endocrinal remission. If signs and symptoms of hypopituitarism were present, replacement therapy was provided. In contrast to hypopituitarism, recurrence was defined as an early remission followed by recurrent hypercortisolism. In addition to these conditions, post-operative complications, including diabetes insipidus, brain infarction and cerebrospinal fluid rhinorrhea were recorded. Persistent rhinorrhea was defined as a long-lasting rhinorrhea for $>7$ days that was not improved following conservative treatment. Persistent hypopituitarism and/or diabetes insipidus were defined based on the requirement for replacement therapy in the first month of the follow-up period. All of the patients were evaluated at $1,3,9$ and 12 months post-operatively, and every 12 months thereafter. Pituitary function and enhanced MRI were also evaluated in the follow-up period.

Statistical analysis. The incidence of endocrinological remission, recurrence and post-operative complications among the patients who underwent inactivation and those without inactivation was compared by Fisher's exact test. All statistical analyses were conducted using GraphPad (version 6.0; GraphPad Software, Inc., La Jolla, CA, USA). P<0.05 indicated that the difference between groups was statistically significant.

\section{Results}

Surgical outcomes. Early endocrinological remission was achieved in 71 cases, while 8 cases exhibited no apparent improvement (Table I). In patients with pre-operative visual impairment, various degrees of visual improvement were noted. In addition, serum PRL returned to normal levels. At 3 months after the operation, the enhanced pituitary MRI scans indicated the following: Gross total resection was achieved in 73 cases and partial resection in 6 cases. Pathological examination indicated pituitary adenoma in 

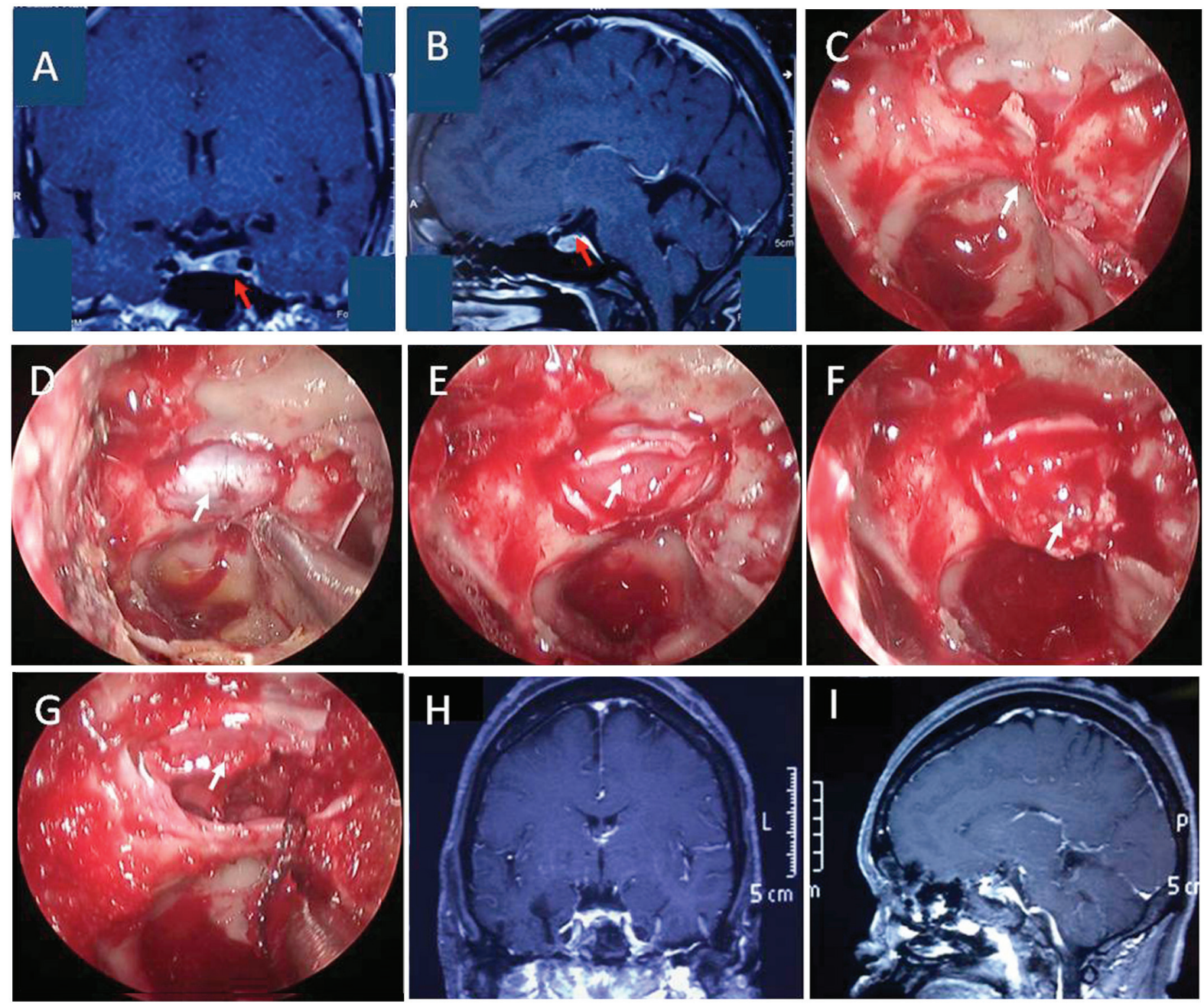

Figure 1. Illustrative case I. A 37-year-old female with Cushing's syndrome also suffered from diabetes mellitus and hypertension for $>3$ years. Her pre-operative high- and low-dose dexamethasone suppression test were negative, and bilateral inferior petrosal sinus sampling revealed that adrenocorticotropic hormone levels in the peripheral blood were $45.8 \pm 2.7 \mathrm{pg} / \mathrm{ml}$ (left inferior petrosal sinus, $351.9 \pm 33.8 \mathrm{pg} / \mathrm{ml}$; right inferior petrosal sinus, $48.1 \pm 1.8 \mathrm{pg} / \mathrm{ml}$ ). (A) Coronal and (B) sagittal views of pre-operative MRI images indicated pituitary microadenoma (red arrows); (C) The ventral wall of the sphenoid sinus was removed and the sellar floor was exposed (white arrow); (D) the bony sellar floor was then drilled and the sellar dura mater was exposed (white arrow); (E) the sellar dura mater was cut and the pituitary tissue was exposed (white arrow); (F) the pituitary gland was explored and white tumor tissues were visualized (white arrow); (G) following gross total resection of the tumor and inactivation of peritumoral pituitary tissue, the diaphragma sellae was exposed as expected. The white arrow indicates the residual normal pituitary tissue. (H) Coronal and (I) sagittal views of enhanced MRI within $24 \mathrm{~h}$ following surgery indicated total resection of the tumor. Scale bar, $5 \mathrm{~cm}$. MRI, magnetic resonance image.

75 cases and normal pituitary tissue in 4 cases, while immunohistochemical analysis revealed positive ACTH staining.

Post-operative complications. Post-operative complications occurred in 18 patients, of whom 7 patients developed persistent symptoms of hypopituitarism, 4 presented with cerebrospinal rhinorrhea, 1 with brain stem infarction and 9 with transient diabetes insipidus (Table II). Three patients simultaneously developed persistent hypopituitarism and transient diabetes insipidus. Regarding complications, no significant differences were identified between patients who underwent inactivation and those who did not. A total of 2 out of the 4 patients with post-operative cerebrospinal fluid rhinorrhea were cured by re-operation, as exemplified in the illustrative case presented in Fig. 3, and the other two were successfully managed by conservative therapy. No cases of confirmed intracranial infection or death associated with the surgical procedures were encountered.
Follow-up. The mean interval of the follow-up was 26 months with a range of 11-62 months. At the time that the follow-up was terminated, all of the 19 patients with post-operative complications were in remission. A total of 4 out of the 7 patients with hypopituitarism who had undergone surgery withdrew from replacement therapy. All of the patients without early remission $(\mathrm{n}=8)$ and all the cases of recurrence $(n=5)$ achieved endocrinological remission following radiotherapy. The enhanced pituitary MRI that was performed during the follow-up period in the 6 patients with partial resection indicated no enlargement of the residual tumor.

\section{Discussion}

Despite the presence of classical clinical symptoms and signs of Cushing's disease, the high-dose dexamethasone suppression test may be negative in certain cases, and the enhanced pituitary MRI scan may fail to indicate the presence of 

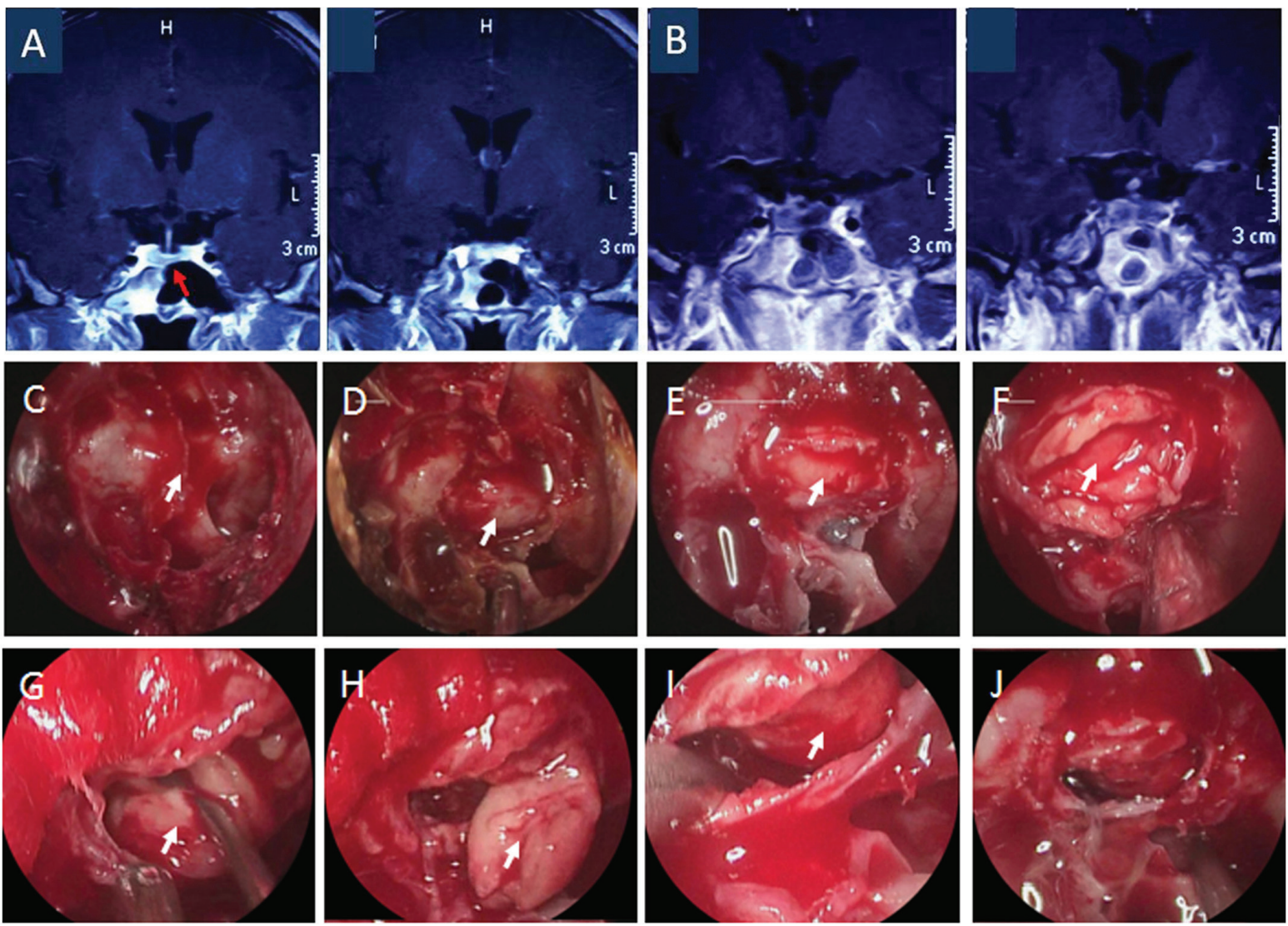

Figure 2. Illustrative case II. A 42-year-old male presented with Cushing's syndrome and diabetes for two years. Pre-operative MRI indicated suspicious signs of tumor. The pre-operative low-dose dexamethasone suppression test was negative, whereas the high-dose dexamethasone suppression test was positive. Bilateral inferior petrosal sinus sampling indicated that the adrenocorticotropic hormone level was $41.6 \pm 7.9 \mathrm{pg} / \mathrm{ml}$ in the peripheral blood, $40.9 \pm 12.5 \mathrm{pg} / \mathrm{ml}$ in the left inferior petrosal sinus and $247.5 \pm 41.0 \mathrm{pg} / \mathrm{ml}$ in the right petrosal sinus. (A) The pre-operative MRI revealed pituitary microadenoma (red arrow); (B) MRI within $24 \mathrm{~h}$ following surgery indicated total resection of the tumor. (C) The ventral wall of the sphenoid sinus was removed and the sellar floor was exposed (white arrow); (D) the bony sellar floor was drilled and the sellar dura mater was exposed (white arrow); (E) the sellar dura mater was cut and the pituitary tissue was visualized (white arrow); (F) the pituitary gland was explored and white tumor tissues was visualized (white arrow); (G and $\mathrm{H}$ ) complete tumor resection was performed along the pseudocapsule. The white arrow indicates the pseudocapsule. (I and J) Following total tumor resection, no residual tumor was detected in the sella turcica and the diaphragm sellae was exposed as expected (white arrow). MRI, magnetic resonance image.

ACTH-secreting adenomas (4,5). Under such circumstances, the BIPSS should be considered $(3,5)$. The pathological results of the present study confirmed the efficiency of this diagnostic strategy. Pituitary surgery with the assistance of endoscopic technology is the preferred option among the treatments for Cushing's disease, as it allows for more accurate visualization of the operative field and reduces blind spots for the surgeon, so as to avoid injury to normal structures $(8,14)$. Hence, the endoscope is able to lay a technical foundation for minimally invasive microneurosurgery within the concept of 'minimal trauma, minimal risk and the greatest degree of lesion resection' (15-17). With the improvement of the neuroimaging techniques and endocrine examinations, the pre-operative diagnostic rate of Cushing's disease has significantly improved, and pre-operative pituitary MRI scans (including plain, enhanced and dynamic enhanced scan) are able to visualize $\sim 80 \%$ of micro-adenomas (18). Despite the possibility of omission, the tumor tissue was successfully localized during exploration in $94.9 \%$ of the patients $(75 / 79)$, even in the 9 of the 13 cases with a negative dynamic MRI scan. The method introduced by Cebula et al (11) was used to explore and localize the adenoma when lesions were not identified on the MRI scans. According to the experience of the authors of the current study, after resecting a macroadenoma that did not have a clear border, patients with a remaining pituitary gland of $<8 \mathrm{~mm}$ had a tendency to go into remission. Hence, it was concluded that those patients require inactivation of peritumoral tissue in order to achieve the highest possible post-operative endocrinal remission rate. Since the location and/or an apparent border of a tumor cannot always be detected, this method was developed to maximize the probability of a curative operation. In our opinion, ETS for Cushing's disease should be performed in high-volume neurosurgical centers with experienced and trained surgeons to reconstruct the sellar floor. By using this surgical method, the tumors may be accessed and endoscopy may be used in order to facilitate sellar floor reconstruction. When using ETS, the authors of the current study worried that cauterization may cause a higher rate of cerebrospinal fluid rhinorrhea. In the present study, a total of 24 patients experienced various degrees of rupture of the diaphragm sellae and arachnoid membrane, while the reconstruction of the sellar floor with autologous fat and hemostatic materials was largely satisfactory. The results suggested that if 
Table I. Clinicopathological characteristics and examination results of the patients $(n=79)$.

\begin{tabular}{|c|c|}
\hline Parameter & Value \\
\hline Age (years) & $44(33-61)$ \\
\hline \multicolumn{2}{|l|}{ Sex } \\
\hline Male & $21(26.6 \%)$ \\
\hline Female & $58(73.4 \%)$ \\
\hline Pre-op cortisol levels $(\mu \mathrm{g} / \mathrm{dl})$ & $33(22.1-43.5)^{\mathrm{a}}$ \\
\hline Pre-op ACTH levels (pg/ml) & $41(26.2-59.1)^{b}$ \\
\hline \multicolumn{2}{|l|}{ High-dose dexamethasone test } \\
\hline Suppressed & $48(60.8 \%)$ \\
\hline Unsuppressed & $16(20.2 \%)$ \\
\hline Bilateral inferior petrosal sinus sampling & $31(39.2 \%)$ \\
\hline No obvious lesion on MRI & $13(16.4 \%)$ \\
\hline $\begin{array}{l}\text { Complications with MRI scan } \\
\text { (Metallic implants) }\end{array}$ & $2(2.5 \%)$ \\
\hline \multicolumn{2}{|l|}{ Pre-op comorbidities } \\
\hline Hypertension & $61(77.2 \%)$ \\
\hline Diabetes mellitus & $47(59.4 \%)$ \\
\hline Transient ischemic attack ${ }^{c}$ & $18(22.8 \%)$ \\
\hline Pituitary hyperplasia & $11(13.9 \%)$ \\
\hline \multicolumn{2}{|l|}{ Tumor size (cm) } \\
\hline$<1$ & $52(65.8 \%)$ \\
\hline$\geq 1$ & $14(17,7 \%)$ \\
\hline $\begin{array}{l}\text { Intra-op rupture of diaphragma sellae } \\
\text { and arachnoid membrane }\end{array}$ & $24(30.4 \%)$ \\
\hline \multicolumn{2}{|l|}{ Extent of tumor resection } \\
\hline Total & $73(92.4 \%)$ \\
\hline Partial & $6(7.6 \%)$ \\
\hline $\begin{array}{l}\text { Inactivation of peritumoral tissue by } \\
\text { cauterization }\end{array}$ & $35(44.3 \%)$ \\
\hline \multicolumn{2}{|l|}{ Pathological examination } \\
\hline Pituitary adenoma & $75(94.9 \%)$ \\
\hline Normal pituitary tissue & $4(5.1 \%)$ \\
\hline \multicolumn{2}{|l|}{ Early endocrinal remission $^{\mathrm{d}}$} \\
\hline Achieved & $71(89.9 \%)$ \\
\hline Stable & $8(10.1 \%)$ \\
\hline Stable disease after partial resection & $6(7.6 \%)$ \\
\hline Recurrence at follow-up ${ }^{\mathrm{e}}$ & $5(6.3 \%)$ \\
\hline Post-op complications & $18(24.1 \%)$ \\
\hline Hypopituitarism & $7(8.9 \%)$ \\
\hline Rhinorrhea & $4(5.1 \%)$ \\
\hline Brainstem infarction & $1(1.3 \%)$ \\
\hline Diabetes insipidus & $9(11.4 \%)$ \\
\hline
\end{tabular}

${ }^{\mathrm{a}}$ Normal range of the cortisol level $(8 \mathrm{am}): 5-20 \mu \mathrm{g} / \mathrm{dl}$. ${ }^{\mathrm{b}}$ Normal range of the ACTH level ( $8 \mathrm{am}): 4.7-48.8 \mathrm{pg} / \mathrm{ml} .{ }^{\mathrm{c}} \geq 1$ attack within 5 years. ${ }^{\text {dEarly }}$ clinical remission was defined as normal fasting plasma cortisol and ACTH levels within the first post-operative

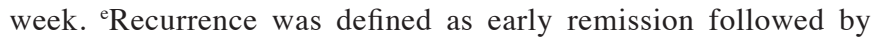
recurrent hypercortisolism at any time during follow up. Values are expressed as $\mathrm{n}(\%)$ or the median (range). Op, operation; $\mathrm{ACTH}$, adrenocorticotropic hormone; MRI, magnetic resonance imaging. surgeons operate meticulously, these ruptures would not lead to major problems, such as cerebrospinal fluid rhinorrhea.

In the early stage of the application of peritumoral activation, two cases of persistent post-operative cerebrospinal fluid rhinorrhea were encountered that were successfully treated by re-operation. To reduce the possibility of post-operative cerebrospinal fluid rhinorrhea, the technical protocol that was followed during the resection of the pituitary ACTH adenoma included the following points: i) Maintenance of a clean surgical field to avoid blind curettage of the tumors. The risk of bleeding during the operation, the major sources of which are intercavernous sinuses, was managed by elevating the upper body to a $20-30^{\circ}$ angle in order to ensure that the head was in a higher position than the heart, thereby reducing venous bleeding. ii) Extrocapsular tumor resections were applied in order to remove pituitary ACTH tumors with pseudocapsule and reduce damage to the normal pituitary tissues, diaphragma sellae and arachnoid membranes. During inactivation of the peritumoral tissue, care was taken to distinguish the pituitary tissue that closely adheres to the diaphragma sellae. Post-operative cerebrospinal fluid rhinorrhea was thereby reduced. iii) Once intra-operative cerebrospinal fluid rhinorrhea occurred, the reconstruction of the diaphragma sellae with fat and/or other autologous tissues was immediately performed at the end of surgery. Sterilization and draping of an alternative abdominal incision prior to ETS is our common practice and may reduce the duration of the surgery. iv) Following surgery, the patient was maintained in an elevated head position, and any postoperative discomforts, including constipation and prostatic hyperplasia, were treated. Furthermore, patients were instructed to avoid sneezing and coughing by force. Post-operative lumbar drainage and antibiotic treatment were applied in cases with high-volume intra-operative cerebrospinal fluid rhinorrhea. In cases of unsuccessful conservative therapy and persistence of rhinorrhea for $>7$ days, re-operation should be considered.

The characteristic manifestation of pituitary ACTH adenoma is Cushing's syndrome. Hypercortisolemia may hinder the body's ability to fight off infection (18). Infections of the central nervous and respiratory systems are particularly prone to occur in these patients (19). Hypertension may lead to instability of vital signs during the surgery, which may cause additional intra-operative bleeding and intracranial hematoma following the operation. High levels of blood glucose prior to and following the surgery may also increase the risk of infection and impair wound healing. In the present study, one case presented with post-operative brain stem infarction, possibly due to a poor condition of the vasculature and surgical stress. Although the patient gradually recovered and returned to work, this case exemplifies the importance of peri-operative management, including fluid balance (as patients can be affected by post-operative diabetes insipidus), blood pressure and glucose control. The multidisciplinary team involved in treating the patients of in the current study was dedicated to the management of the surgery of the functional pituitary adenoma. At the beginning, the majority of patients enrolled in the present study were admitted to the Department of Endocrinology of Qilu Hospital of Shandong University (Jinan, China). 
Table II. Association between the cauterization of peritumoral tissue and outcomes/complications.

\begin{tabular}{|c|c|c|c|}
\hline Item & Inactivation $(n=35)(\%)$ & No inactivation $(n=44)(\%)$ & P-value \\
\hline \multicolumn{4}{|l|}{ Outcome } \\
\hline Early remission $^{\mathrm{a}}$ & $30(38.0)$ & $41(51.9)$ & 0.2744 \\
\hline Recurrence $^{\mathrm{b}}$ & $2(2.5)$ & $3(3.8)$ & 0.9157 \\
\hline \multicolumn{4}{|l|}{ Complications } \\
\hline Diabetes insipidus & $6(7.6)$ & $3(3.8)$ & 0.1743 \\
\hline Persistent hypopituitarism & $5(6.3)$ & $2(2.5)$ & 0.2314 \\
\hline $\begin{array}{l}\text { Concomitant diabetes insipidus and } \\
\text { persistent hypopituitarism }\end{array}$ & $2(2.5)$ & $1(1.3)$ & 0.4266 \\
\hline $\begin{array}{l}\text { Intraoperative rupture of diaphragma } \\
\text { sellae and arachnoid membrane }\end{array}$ & $13(16.5)$ & $11(13.9)$ & 0.3255 \\
\hline Rhinorrhea & $2(2.5)$ & $2(2.5)$ & 1.0000 \\
\hline
\end{tabular}

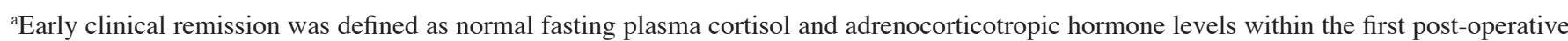
week. 'Recurrence was defined as early remission followed by recurrent hypercortisolism at any time during follow up. Patients with concomitant diabetes insipidus and persistent hypopituitarism are also included in the diabetes insipidus and persistent hypopituitarism groups.
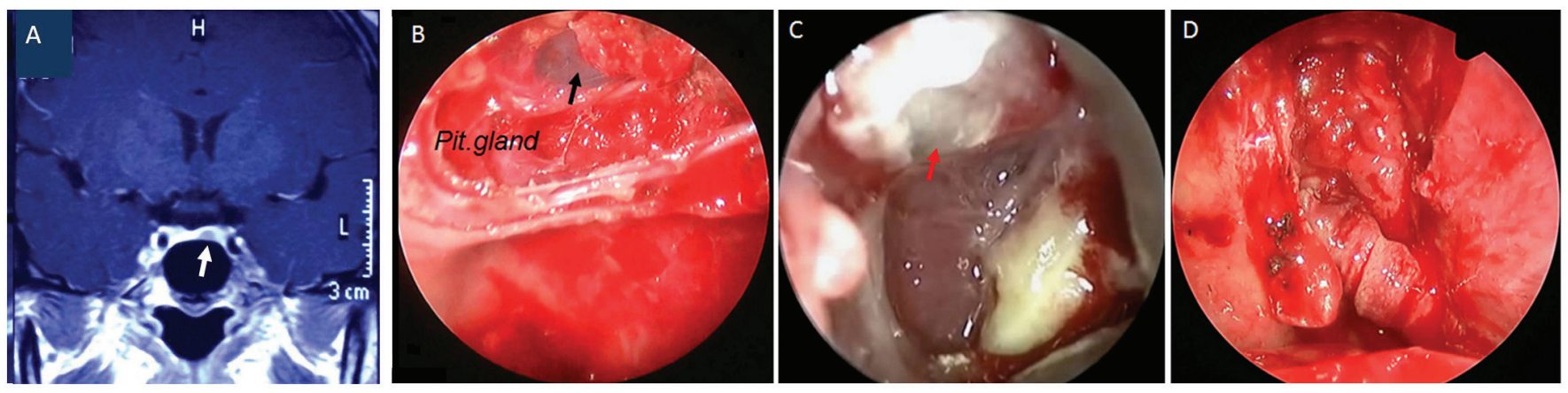

Figure 3. Illustrative case III. A 37-year-old female had a history of Cushing's syndrome for 5 years and gradual visual loss for 1 year. (A) Pre-operative magnetic resonance image revealed pituitary microadenoma (white arrow). (B) Following total resection of the tumor, normal pituitary tissue (indicated as 'Pit.gland') and a rupture of the arachnoid membrane (black arrow) were observed. The patient did not present with rhinorrhea immediately after surgery and was discharged. (C) At 1 month following surgery, the patient was re-admitted to the hospital due to persistent rhinorrhea ( $\sim 15$ days despite bed rest). An endoscopic examination was performed immediately. The fistula (red arrow) was visualized under local anesthesia. (D) A nasal septal flap was harvested and used to reconstruct the sellar floor. The rhinorrhea was cured following re-operation.

All of the patients were subjected to endocrinal examinations and comprehensive assessments were performed by endocrinologists. Subsequently, the expert team, consisting of experienced neurosurgeons, endocrinologists, anesthesiologists, radiologists and pathologists, reviewed the medical records of each of the patients and derived an individualized protocol. In terms of remission, it should be kept in mind that the outcomes across various studies are different depending on the criteria used to define remission and the timing of evaluation. A recent surgical series revealed remission rates of $65-85 \%$ and recurrence rates of $10-35 \%$ (1). With regard to the patients with recurrent post-operative Cushing's syndrome, radiotherapy was able to attain endocrinological remission.

In conclusion, although limited by its retrospective nature, the present study included a relatively large population of 79 patients with Cushing's disease. The overall post-operative remission rate was $83.5 \%(66 / 79)$, which is comparable to that of other studies $(1,2)$. Overall, compared with conventional ETS procedures, the data suggest that inactivation of peritumoral tissue is a comparable method in the treatment of intricate Cushing's disease in terms of efficacy and safety. However, the present results require further verification by large prospective studies.

\section{Acknowledgements}

The authors would like to thank Dr Hongwei Qin (Department of Cell, Development, and Integrative Biology, University of Alabama at Birmingham, Birmingham, AL, USA) for revising this manuscript.

\section{Funding}

This work was supported by the National Natural Science Foundation of China (grant no. 81201986) and the foundation for outstanding young and middle-aged scientists of Shandong province (grant no. BS2013YY018). 


\section{Availability of data and materials}

The datasets used and/or analyzed during the current study are available from the corresponding author on reasonable request.

\section{Authors' contributions}

$\mathrm{SC}$ and FLin were major contributors in revising the manuscript as well as analyzing and interpreting the data. SX was the major surgeon performing ETS. XZ made substantial contributions to acquisition of the data and the preparation of the manuscript. MD and FLiu performed the pre-operative endocrinal examinations and assessment. XL and XM made substantial contributions to the conception and design and supervised the study. All authors read and approved the final manuscript.

\section{Ethics approval and consent to participate}

This study was approved by the Ethics Committee of Qilu Hospital (Jinan, China) and written informed consent was obtained from all patients.

\section{Patient consent for publication}

Written informed consent and/or oral consent was obtained from all included patients and/or their relatives for publication of associated images.

\section{Competing interests}

The authors declare that they have no competing interests.

\section{References}

1. Lonser RR, Nieman L and Oldfield EH: Cushing's disease: Pathobiology, diagnosis, and management. J Neurosurg 126: 404-417, 2017.

2. Molitch ME: Diagnosis and treatment of pituitary adenomas: A review. JAMA 317: 516-524, 2017.

3. Esposito V, Santoro A, Minniti G, Salvati M, Innocenzi G, Lanzetta $\mathrm{G}$ and Cantore $\mathrm{G}$ : Transsphenoidal adenomectomy for GH-, PRL- and ACTH-secreting pituitary tumours: Outcome analysis in a series of 125 patients. Neurol Sci 25: 251-256, 2004.

4. Hardy J: Transphenoidal microsurgery of the normal and pathological pituitary. Clin Neurosurg 16: 185-217, 1969.

5. Mehta GU, Lonser RR and Oldfield EH: The history of pituitary surgery for Cushing disease. J Neurosurg 116: 261-268, 2012.
6. Dehdashti AR, Ganna A, Karabatsou K and Gentili F: Pure endoscopic endonasal approach for pituitary adenomas: Early surgical results in 200 patients and comparison with previous microsurgical series. Neurosurgery 62: 1006-1015, 2008.

7. Frank G, Pasquini E, Farneti G, Mazzatenta D, Sciarretta V, Grasso V and Faustini Fustini M: The endoscopic versus the traditional approach in pituitary surgery. Neuroendocrinology 83: 240-248, 2006.

8. Gondim JA, Schops M, de Almeida JP, de Albuquerque LA, Gomes E, Ferraz T and Barroso FA: Endoscopic endonasal transsphenoidal surgery: Surgical results of 228 pituitary adenomas treated in a pituitary center. Pituitary 13: 68-77, 2010.

9. Kabil MS, Eby JB and Shahinian HK: Fully endoscopic endonasal vs. transseptal transsphenoidal pituitary surgery. Minim Invasive Neurosurg 48: 348-354, 2005.

10. Pivonello R, Isidori AM, De Martino MC, Newell-Price J, Biller BM and Colao A: Complications of Cushing's syndrome: State of the art. Lancet Diabetes Endocrinol 4: 611-629, 2016.

11. Cebula H, Baussart B, Villa C, Assié G, Boulin A, Foubert L, Aldea S, Bennis S, Bernier M, Proust F and Gaillard S: Efficacy of endoscopic endonasal transsphenoidal surgery for Cushing's disease in 230 patients with positive and negative MRI. Acta Neurochir (Wien) 159: 1227-1236, 2017.

12. Starke RM, Reames DL, Chen CJ, Laws ER and Jane JA Jr: Endoscopic transsphenoidal surgery for cushing disease: Techniques, outcomes, and predictors of remission. Neurosurgery 72: 240-247, 2013.

13. Lopes MBS: The 2017 World Health Organization classification of tumors of the pituitary gland: A summary. Acta Neuropathol 134: 521-535, 2017.

14. Goudakos JK, Markou KD and Georgalas C: Endoscopic versus microscopic trans-sphenoidal pituitary surgery: A systematic review and meta-analysis. Clin Otolaryngol 36: 212-220, 2011.

15. Ammirati M, Wei L and Ciric I: Short-term outcome of endoscopic versus microscopic pituitary adenoma surgery: A systematic review and meta-analysis. J Neurol Neurosurg Psychiatry 84: 843-849, 2013.

16. Tabaee A, Anand VK, Barrón Y, Hiltzik DH, Brown SM, Kacker A, Mazumdar M and Schwartz TH: Endoscopic pituitary surgery: A systematic review and meta-analysis. J Neurosurg 111: 545-554, 2009.

17. Sarkar S, Rajaratnam S, Chacko G, Mani S, Hesargatta AS and Chacko AG: Pure endoscopic transsphenoidal surgery for functional pituitary adenomas: Outcomes with Cushing's disease. Acta Neurochir (Wien) 158: 77-86, 2016.

18. Hofstetter CP, Shin BJ, Mubita L, Huang C, Anand VK, Boockvar JA and Schwartz TH: Endoscopic endonasal transsphenoidal surgery for functional pituitary adenomas. Neurosurg Focus 30: E10, 2011.

19. Choe JH, Lee KS, Jeun SS, Cho JH and Hong YK: Endocrine outcome of endoscopic endonasal transsphenoidal surgery in functioning pituitary adenomas. J Korean Neurosurg Soc 44: $151-155,2008$.

This work is licensed under a Creative Commons Attribution-NonCommercial-NoDerivatives 4.0 International (CC BY-NC-ND 4.0) License. 